\title{
Der Raum des Sertão und Literatur: Das Rosianische Pendel
}

\section{Vorausgehende Überlegungen}

Diese Studie zielt darauf ab, einige Aspekte in Bezug auf die Darstellung des Raums des Sertão in der Literatur von João Guimarães Rosa zu untersuchen. Da die Interaktionen des Schriftstellers mit dem Raum des Sertão entscheidend für die Komposition seiner Werke waren, wird herausgearbeitet, wie seine Literatur gleich der Bewegung eines Pendels auf verschiedene Weise in dem Raum nachhallt, der ihr vielfältige Anreize gab.

Einige persönliche Erfahrungen, die durch Rosas Reisen in den Campos Gerais ermöglicht wurden, welche er mit der Absicht antrat, die dortige soziokulturelle Dynamik zu erforschen und von denen die Reise aus dem Jahre $1952^{1}$ besonders hervorzuheben ist, fungierten als erste Dialoge eines diskursiven Netzwerks, in das sich seine literarische Produktion einfügt. Dies realisiert sich vor allem in der Interaktion mit den Lesern und dient gleichzeitig als Anknüpfungspunkt für die ersten Erfahrungen des Künstlers und als Impuls für einen soziokulturellen Austausch, in der Bedeutungszusammenhänge neu verhandelt werden. Bevor wir jedoch derartige Überlegungen anstellen, soll vorangehend angemerkt werden, dass nicht die literarische Produktion von Guimarães Rosa als dem Schöpfer des kulturellen Reichtums der Region des Sertão im Zentrum der Untersuchung liegt. Ein solcher kultureller Reichtum der Literatur des Schriftstellers wird hier zweifelsfrei vorausgesetzt. Als Folge der Entscheidung des Künstlers, sein literarisches Werk - aufgrund der Bewunderung und Anerkennung ihrer Größe - mit dem Raum und der Kultur des Sertão als Hauptreferenzen zu kreieren, zeigt seine Produktion Spuren des Beitrags zur Aufwertung und Verbreitung dieser Kultur. In einem solchen Sinne diskutiert diese Studie, inwiefern Rosas Literatur ein gewisses Potenzial für die Entwicklung von Initiativen zu Förderung und

1 Für das ausführliche Studium dieser vom Autor durchgeführten Reise wurde sein Notizbuch seiner ursprünglichen Reise, sowie die maschinenschriftlichen Abschriften desselben in Forschungen konsultiert, die in den Archiven des Instituts für Brasilianische Studien durchgeführt wurden (Instituto de Estudos Brasileiros, IEB-USP) in São Paulo sind. Vgl. (IEB-USP-Archiv, Sammlung João Guimarães Rosa, HEFT-06, Box 082); (IEB-USP-Archiv, Sammlung João Guimarães Rosa, EO-13.01, Box 073, A boiada. Band 1 und Band 2).

Ә Open Access. (C 2020 Fabrício César de Aguiar, publiziert von De Gruyter. (c) BY-NC-ND Dieses Werk ist lizenziert unter der Creative Commons Attribution-NonCommercial-NoDerivatives 4.0 Lizenz. https://doi.org/10.1515/9783110677713-010 
Stimulation eines Dynamikwandels von Raum und Kultur des Sertão hervorbringt.

\section{Die rosianische Literatur: angeregt von und Anreger der Kultur des Sertão}

In den letzten Jahrzehnten lenkt die rosianische Literatur die Aufmerksamkeit auf die Kultur und die soziale Realität des Landes, die auf vielfältige Art und Weise von einer komplexen Dynamik gekennzeichnet ist.

Dies lässt sich unter anderem in der Zahl der Interessierten erkennen, die, angeregt vom Kontakt mit den Werken des aus Minas stammenden Schriftstellers, begannen, ihre Aufmerksamkeit auf die vom Schriftsteller als Referenz für seine Literatur benutzte Region zu richten und Forschungfragen aufzuwerfen. Es existieren bereits Studien, die auf den von Wissenschasftlern durchgeführten Expeditionen basieren. Hier wären beispielsweise folgende zu nennen: Alan Viggiano ${ }^{2}$, der versucht, die Route Riobaldos ausfindig zu machen; Marily da Cunha Bezerra und Dieter Heidermann ${ }^{3}$, die Reisen zur Erschließung der Räume unternahmen, auf die in der Literatur Rosas verwiesen wird; Willi Bolle, der nach mehreren Reisen die Kartierung der Bewegungen der Jagunços in Grande sertão: veredas ${ }^{4}$ minutiös erarbeitet.

In meinem Fall wurden zwei Reisen mit dem Ziel durchgeführt, Feldstudien über einen Ausschnitt der immensen Weite des Sertão und einen Teil seiner Kultur voranzutreiben. Die erste fand vom 02. bis 10. Juli 2016 unter Einbindung einer ausgewählten Teilnehmergruppe in der 3. Auflage des Projektes «O Caminho do Sertão» (dt. «Der Weg des Sertão») statt. Begleitet von Menschen aus verschiedenen Regionen Brasiliens, geführt durch weise Bewohner der Region mit erkennbaren Ähnlichkeiten zur literarischen Figur des Pedro Orósio und aufgenommen

2 Vgl. Alan Viggiano: Itinerário de Riobaldo Tatarana. In: Antônio Carlos Secchin u.a. (Hg.): Veredas no sertão rosiano. Rio de Janeiro: 7Letras 2007, S. 85-102.

3 Vgl. Marily da Cunha Bezzera/Dieter Heidermann: Viajar pelo sertão roseano é antes de tudo uma descoberta. In: Revista Estudos Avançados - USP 20/58 (2006): Dossiê Guimarães Rosa, S. 7-17.

4 Der Wissenschaftler entwickelt anhand seiner Feldstudien eine Karte, die die realen Räume des Sertão mit dem fiktionalen Raum von Grande sertão: veredas in Beziehung setzt, sowie drei weitere Karten, die «Topographie der Jagunçerei» genannt werden und die Verschiebungen der Jagunços auf dem Spielbrett des rosianischen Raums beschreiben. Vgl. Willi Bolle: grandesertão.br: o romance de formação do Brasil. São Paulo: Duas Cidades/Editora 34 2004, S. 69, S. 103, S. 109, S. 115 . 
von der unbeschreiblichen Gastfreundschaft der «Mineiros» gingen wir etwa 170 km entlang der Nordwest-Region von Minas Gerais und interagierten dabei mit Umwelt, Kultur und den verschiedenen Aspekten der sozialen Realität der Region. Ausgangspunkt war das Dorf Vila de Sagarana. Nach Überquerung des Flusses Urucúia passierten wir Morrinhos, Vila Bom Jesus, Fazenda Menino, Córrego Garimpeiro, Ribeirão de Areia, Buraquinhos, Vão dos Buracos und die Gemeinde der Chapada Gaúcha, mit dem Nationalpark Grande Sertão Veredas als Zielpunkt der Wanderung, der sich bereits an der Grenze zum Bundesstaat Bahia befindet. Für eine gewisse Veranschaulichung des durchaufenen Wegs in dieser Travessia (dt. Durchquerung), siehe Karte 4 im Beitrag von Willi Bolle (S. 78).

Gleich der Figur Grivo aus dem Text Cara-de-Bronze, begeben wir uns auf die Suche nach dem wer der Dinge: «Zu sehen, zu hören und zu fühlen. Und zu wählen. Seine Augen wurden nicht müde. ${ }^{5}$ Solche Worte stellen die Erfahrung der Wanderung gut dar. Ein derartiges Eintauchen in dieses Stück mineirischen Sertão war so prägend, dass es für ein Umdenken von verschiedenen Aspekten aus Guimarães Rosas Werk und dieser Region des Sertão sorgte. Begeistert von den zahlreichen Entdeckungen und mit der Bestrebung, die Beziehung der rosianischen Literatur mit dem mineirischen Sertão detaillierter zu erforschen - sowohl als Frucht der Anregungen durch die Erfahrungen des Autors also auch hinsichtlich seines Charakters als aktuell potenzialschöpfender Kraft für soziokulturellen Wandel in diversen Bereichen - reiste ich vom 23. bis 25. Januar 2017 wieder in die Region zurück. Meine Absicht war, einige Interviews mit Bewohnern, sozialen Führern, Organisatoren und Partnern diverser repräsentativer kultureller Projekte durchzuführen sowie um Daten und Zeugnisse über Agenturen und Institute zu sammeln, die mit der ganzheitlichen und nachhaltigen Entwicklung der Region zusammenhängen. Das spezifische Interesse an der Sammlung und Auswahl von Materialien ergab sich während der Recherchen zu Initiativen, die in irgendeiner Weise mit Aktionen in Zusammenhang standen, die auf dem in der Region aufgrund der rosianischen Literatur entstandenen Potenzial beruhten. $\mathrm{Zu}$ diesem Zweck und im Bewusstsein des immensen Territoriums des Sertão in Minas Gerais/Goiás/Bahia konzentrierte ich mich auf die nordwestliche Region von Minas Gerais, eine Region des Urucuia-Beckens, das Start- und Zielpunkt der Travessia von 2016 umfasste.

Man konnte erkennen, dass der Rio Urucuia, der als Riobaldos «Fluss der Liebe» repräsentativ für Rosas literarische Schöpfung ist, für die gesamte natürliche

5 Guimarães Rosa: Corpo de Baile. Bd. 2. Rio de Janeiro: Nova Fronteira 2010b, S. 258. Üb. Jõao Guimarães Rosa: Grande Sertão. Aus dem brasilianischen Portugiesisch von Curt Meyer-Clason. Köln: Kiepenheuer \& Witsch ${ }^{3} 1994$, S. 578. 
und soziale Dynamik der Region von grundlegender Bedeutung ist. Laut Rosa Amélia Pereira da Silva war

das Vale do Urucuia aufgrund seiner großen territorialen Reichweite ein strategischer Punkt: ein Ort der Konvergenz zwischen Viehtreibern und Viehzüchtern, Minenarbeitern, Entdeckern und Abenteurern, die im Landesinneren Brasiliens eine Bereicherung sowohl für die Suche und den Abbau von Mineralien als auch für die Niederlassung in den Regionen suchten, mit der Absicht, Landwirtschaft in noch nicht beherrschten Gebieten zu betreiben. ${ }^{6}$

Die Forscherin weist darauf hin, dass «der Rio Urucuia ein Akteur in der sozialen Dynamik ist; er fördert Vereinigungen und Aggregationen, die die Gesellschaften konstituieren, denn um ihn herum entstand und entsteht noch immer eine kulturelle Bewegung.» ${ }^{7}$ In ihrer Arbeit interpretiert sie die Beziehungen zwischen diesem Fluss und der rosianischen Literatur, die als Akteure einer geo-soziokulturellen Dynamik die Region gestalten. Laut der Wissenschaftlerin sind «der Fluss und JGR [João Guimarães Rosa] in der sozialen Formation des Rio Urucuia-Tals wie in der geographischen, wirtschaftlichen, politischen und kulturellen Dynamik Vermittler im Prozess der Gruppenwiedervereinigung». ${ }^{8}$

Rosas literarische Produktion, die auf einzigartige Weise die verschiedensten sozio-kulturellen Aspekte dieser Region repräsentiert und ihnen nationale und internationale Sichtbarkeit verliehen hat, hat dazu beigetragen, dass Institutionen, öffentliche Stellen sowie die lokalen Bewohner selbst oder Menschen aus den abgelegensten Teilen des Landes und soziale Gruppen mit den unterschiedlichsten Interessen ihre Augen auf die Region richten. Ihre Reichtümer werden anerkannt und soziale und ökologische Missstände werden ins Auge gefasst. Alle Aspekte, die in vielerlei Hinsicht soziale, wirtschaftliche, kulturelle und ökologische Veränderungen auf verschiedenen Ebenen hervorgebracht haben, stehen nun im

6 Die Forscherin beschreibt, dass «der Fluss Urucuia durch ein Entwässerungsgebiet von 25.135 $\mathrm{km}^{2}$ fließt; es handelt sich um das viertgrößte Becken im Bundesstaat Minas Gerais mit seiner Quelle im Bundesstaat Goiás an der Pouso Alto in der Gemeinde Formosa. Das Urucuia-Tal umfasst derzeit 11 Gemeinden, von denen zehn im Bundesstaat Minas Gerais liegen und den Nordwesten sowie den Norden von Minas Gerais durchqueren: Auf der linken Seite des Flusses liegen die Gemeinden Formoso (1962), Arinos (1962), Chapada Gaúcha (1996) Urucuia (1996) und Pintópolis (1996); auf der rechten Seite Buritis (1962), Uruana de Minas (1996), Riachinho (1996), Bonfinópolis de Minas (1962) und São Romão (1923). Der Fluss zieht eine Grenze zwischen den Gemeinden Pintópolis und São Romão und fließt dort in den São Francisco.» Vgl. Rosa Amélia Pereira da Silva: Nesta água que não para: leitura de João Guimarães Rosa no Vale do Urucuia. [dt. In diesem nie versiegenden Wasser: Lektüre von João Guimarães Rosa im Urucuia-Tal] Brasília: Editora UnB 2014, S. 24.

7 Silva: Nesta água que não para, S. 25.

8 Ebda. 
Zentrum der Auseinandersetzung mit dieser Region. Laut der Forscherin «entstehen um den Namen JGR eine Reihe von Vereinigungen, die kollektive, humanitäre, wirtschaftliche und ökologische Interessen haben.» ${ }^{9}$

In diesem Sinne ist ein für solche Fragen herausstechender Ort in der Region das Dorf Vila Sagarana, ein Ort, der nach dem gleichnamigen literarischen Werk von Guimarães Rosa benannt wurde, dem Ausgangspunkt der Wanderung des Schriftstellers durch die literarische Prosa. Wie der Schriftsteller im Jahre 1966 in einem Interview mit Fernando Camacho berichtete: «im Grunde gab es Sagara$n a^{10}$, um den Leuten Minas zu zeigen. Eine Mischung: Minas zeigen und demonstrieren, wie ich die Welt sah». ${ }^{11}$ Fakt ist, dass dieser Versuch erfolgreich war und sich auf die folgenden Arbeiten ausdehnte. Das Dorf Sagarana, Ausgangspunkt des Projektes «O Caminho do Sertão», profiliert sich derzeit im Nordwesten von Minas Gerais als Schlüsselort für Erhalt, Förderung und Aufwertung der sertanejo-Kultur. Es entsteht aus dem «Segundo Assentamento da Reforma Agrária implantado pelo INCRA Minas Gerais, em 1973» (dt. «der Zweiten Besiedlung der Agrarreform, 1973 errichtet von INCRA Minas Gerais»). ${ }^{12}$ Die Beziehung dieses Raumes zur rosianischen Literatur, die schon vor der Entstehung des Dorfes vorhanden war, ist untrennbar. Laut Silva bekam die Siedlung diesen Namen, ${ }^{13}$ denn

nachdem sie die vor allem räumliche Beziehung zwischen dem Fluss Urucuia und der Fazenda Boi Preto (Schwarzer Bulle) ermittelt und die existierende literarische Beziehung zwischen dem Fluss Urucuia und JGR anerkannt hatten, machten die fachlichen Verantwortlichen für das Projekt in der Region den Vorschlag, dass die Siedlung den Namen eines Werkes des Autors tragen sollte: Sagarana. Das «Projeto Integrado de Colonização de Sagarana - PICS» (dt. «Integratives Projekt der Kolonisation von Sagarana») war geboren.

Wie Almir Paraka Cristóvão Cardoso ${ }^{14}$ in einem Interview vor Ort am 23.1.2017 kommentiert, beschlossen die Fachkräfte von INCRA im Bewusstsein der räumli-

9 Ebda., S. 58.

10 Die Arbeit wird mit dem Neologismus betitelt, der «durch die ausdrucksvolle Suffixierung der nordischen Vokabel Saga mit der im Tupi-Guarani Kollektive anzeigenden Substantiv-Endung «-rana» konstruiert wird.»Vilma Guimarães Rosa: Relembramentos: João Guimarães Rosa, meu pai. Rio de Janeiro: Nova Fronteira 2008, S. 54. Unsere Übersetzung, Anm. d. Üb.

11 Fernando Camacho: Entrevista com João Guimarães Rosa. In: Revista Humboldt 18/37 (1978), S. 42-53, hier S. 45. Unsere Übersetzung, Anm. d. Üb.

12 Verfügbar unter https://festivalsagarana.wordpress.com/o-festival/. Letzter Zugriff: 30.1. 2018.

13 Silva: Nesta água que não para, S. 46.

14 Der «Sohn der Stadt Paracatú» und «Enkel eines Urucuiano», wie er im Interview betont, hat einen sehr wichtigen Landsmann: «Riobaldo selbst, der im Quellgebiet des großen Grüns von Pa- 
chen und kulturellen Beziehung der Region zur rosianischen Literatur, die Siedlung mit diesem speziellen Namen aus dem Werk des Schriftstellers zu benennen, weil

sie auf einer Rückreise nach Belo Horizonte, wo INCRA operierte, nach Cordisburgo hineinfuhren und im Museum Guimarães Rosa, das bereits Anfang der 70er Jahre existierte, das ausgestellte Werk fanden. Dann rief einer der Techniker die anderen und sagte: «Hier ist der Name, nach dem wir für die Siedlung gesucht haben». ${ }^{15}$

Als Bezirk der Gemeinde Arinos ${ }^{16}$ befindet sich Sagarana im Flusseinzugsgebiet des Flusses Urucuia, ein «durch großen sozialen Widerspruch zwischen der grausamen unternehmerischen Landwirtschaft und der großen Zahl von Siedlungen der Agrarreform und traditionellen bäuerlichen Familien geprägter Raum». ${ }^{17}$ Im Format von Agrovilas ${ }^{18}$ umgesetzt ist das Dorf ein wichtiger Widerstandspunkt gegen das räuberische Modell der ausbeutenden Handhabe der Umwelt, wie es von den Großgrundbesitzern des Agrobusiness in der Region betrieben wird. ${ }^{19}$ Laut Silva «besteht heute eine inhärente Beziehung zwischen ihm, JGR, und dem geo-

racatú geboren wurde. Eigentlich vom kleinen Grün von Paracatú, wo heute die Gemeinde von João Pinheiro liegt, zu jener Zeit Gemeinde Paracatú». Der ehemalige Bürgermeister der Gemeinde Paracatú, ehemaliger Abgeordneter der Provinz MG, ist einer der großen Verantwortlichen für die planmäßigen ökologisch-soziokulturellen Veränderungen in der Region Sagarana, die insbesondere auf dem «immateriellen Erbe» des Schriftstellers von Minas Gerais basieren.

15 Auszug aus dem Interview, das am 23.1.2017 im Sertão von Minas Gerais gegeben wurde.

16 Wie Silva über das Urucuia-Flusstal betont, «wählte die Bevölkerung mit der Entwicklung der Region Barra da Vaca zum Bezirkssitz, was Conceição de Morrinhos dieses Privileg entzog. 1962 emanzipierte sich das Dörfchen und wurde zur unabhängigen Stadt. Sie erhielt den Namen Arinos zu Ehren von Afonso Arinos de Melo Franco, einem in Paracatú geborenen Professor für Geschichte und berühmten Literaten aus Minas, einem Pionier in den regionalistischen Tendenzen der brasilianischen Literatur, für die Ausrichtung seiner Arbeit, die aus Erfahrungen im Kontakt mit dem Sertão resultierte». Vgl. Silva: Nesta água que não para, S. 28.

17 Verfügbar unter https://festivalsagarana.wordpress.com/o-festival/. Letzter Zugriff: 30.1.2018 (unsere Übersetzung, Anm. d. Üb.).

18 Wie Almir Paraka im Interview kommentiert, «hatten die Siedler im Modell der Agrovila, das in der Siedlung der Agrarreform errichtet worden war, Grundstücke und Wohnungen im Städtchen und Fläche zum bearbeiten im ländlichen Raum. (Bei Agrovila handelt es sich um eine Art Vorstadtsiedlung, die an das infrastrukturelle Versorgungsnetz einer größeren Stadt angebunden ist und gleichzeitig das ländliche Wohnen ermöglicht, Anm. d.Üb.).

19 Während des Interviews betont Almir Paraka, dass «die Siedlung von Sagarana nicht die Frucht dieser aktuelleren Kämpfe um Boden ist. Hier haben wir Märtyrer, die Widerstand geleistet haben, berühmte Märtyrer, die sich dem Coronelismo widersetzten, die einer ganzen konservativen Offensive gegenüberstanden, um das Recht der Kleinbauern, der Sertanejos, ihr Stück Land zu erhalten.» (Coronelismo: beschreibt die archaisch- patriachalischen Machtstrukturen im Sertão, wo der Großgrundbesitzer, der «coronel» durch Gebrauch seiner wirtschaftlichen Macht und 
graphischen Raum, dessen Bande zur Institutionalisierung des Namens des Schriftstellers in der Position eines wichtigen Akteurs beitragen.» ${ }^{20}$ Im Bewusstsein dieser in der kollektiven Vorstellung der brasilianischen Kultur und insbesondere in der Region des Urucuia erreichten Position bemerkt Almir Paraka, dass in Bezug auf mehrere Projekte für die Entwicklung der Region «Rosas Werk aus einer nachhaltigen, integrierten, territorialen Sichtweise heraus neben den anderen existierenden ein sehr wichtiges immaterielles Erbe in dieser Region ist». ${ }^{21}$ In Bezug auf die Erkennung des Potenzials dieses «immateriellen Erbes» als fruchtbarem Boden für die Aussaat von Projekten, die auf sozio-kulturellen Wandel ausgerichtet sind, der die Lebensbedingungen der Bewohner verbessert und die lokale Kultur aufwertet, kommentiert er zu einem anderen Zeitpunkt des Interviews:

Ich habe in Rosas Werk eine sehr bedeutende Identität gefunden [...] eine künstlerisch-kulturelle Identität, dadurch, dass ausgehend von der Literatur, von Rosas Konzeption, von der Rolle von Kunst und Kultur der Wandel der Personen und der Gesellschaft stattfand. Sein Weg der Transformation ist ein künstlerisch-kultureller Weg und eine zeitlich-räumliche Identität des Sertão. [...] [Dies] ist Rosas Ort, der Ort von Rosas Werk, und darüber hinaus ist es auch ein wenig ein kognitives Erbe, viel mehr als bloß kulturell, ein Erbe nicht nur von statischen und angehäuften Kenntnissen, ein Erbe, das mit der Konstruktion von Wissen verbunden ist, mit der Wahrnehmung der Bedeutung von Wissen und wie Wissen gemacht wird, wie es sich darbietet. Damit verbunden wurden das lokale, traditionelle sowie das Wissen der Personen, die seit jeher dort gewohnt haben, von Rosa porträtiert und noch heute sind sie Erben dieses kulturellen Erbes von Brasiliens Landesinnerem, das Rosa in seinen Werken bestens eingefangen und dokumentiert hat. ${ }^{22}$

Unter dieser Perspektive zeigt sich in der Anerkennung dieses kulturellen Erbes und seines Potenzials, basierend auf den in der Region durchgeführten Aktionen, die Untrennbarkeit der kulturellen Aspekte und der Umwelt als wichtigsten Vermittlern der wesentlichsten Veränderungen in der Region. Wie Silva in Bezug auf den Rio Urucuia und das Werk von João Guimarães Rosa kommentiert «agiert ersterer geo-historisch und wirtschaftlich in der Region. Zweiteres handelt aus zwei Positionen heraus: die eine politisch und die andere literarisch». ${ }^{23}$ Daher kommentiert Almir Paraka bezüglich der ersten entscheidenden Bewegungen in der

durch Gewalt Einfluss auf die dortige Bevölkerung nimmt und zu deren Referenz wird, z.B. in politischen oder in Streitfragen, Anm. d. Üb.).

20 Silva: Nesta água que não para, S. 20.

21 Auszug aus dem von Almir Paraka gegebenen Interview am 23.1.2017 im Sertão von Minas Gerais.

22 Almir Paraka: wie Anm. 21.

23 Silva: Nesta água que não para, S. 20. 
Region, um die laufenden Projekte im Zusammenhang mit diesen beiden Vermittlern zum Laufen zu bringen:

Die zentrale Motivation ist, dass um die Jahrtausendwende eine Gruppe von Führungskräften hier in der Region anfing, Vorschläge für interkommunale Zusammenarbeit als eine die Region denkende Zusammenarbeit zwischen den Gemeinden zu diskutieren, wie man Kräfte zusammenbringen kann, um Lösungen für die verschiedensten die Region betreffende Probleme bezüglich der Lebensqualität der Gemeindebürger in die Wege zu leiten. Diese Ideen bewegten sich um eine zentrale Idee, die wir als nachhaltige und integrierte regionale oder territoriale Entwicklung definieren. Wir definieren das Territorium als ein Flusseinzugsgebiet, um all diese Dimensionen von Wasser und Nachhaltigkeit in den Mittelpunkt der Diskussion zu stellen, also als Territorium, das durch das Becken des Urucuia Flussgebietes definiert wird, und auf diesem Wege zunächst zu mobilisieren, zu sensibilisieren, die verschiedensten Sektoren und sozialen Bereiche zu organisieren, so dass sie selbst auf die größtmögliche autonome Art und Weise Verantwortung im Veränderungs- oder Wandlungsprozess übernehmen können, an den sie glauben, wie sie sagten bzw. sagen. In diesem Bemühen steckt also eine grundlegend notwendige Arbeit, und zwar die Anerkennung, dass jedes Territorium in erster Linie ein kulturelles Territorium ist. Wenn es also kulturell ist, ist es so, als würde man diese kulturelle Dimension zugunsten des gewünschten Wandlungsprozesses bearbeiten, und dort entsteht die Vorstellung von Identität, diese Verbindung mit dem Ort, dem Territorium, den Lebensweisen etc. und diese andere Dimension, verbunden mit jener Individualität, die das Selbstwertgefühl betrifft. Du musst dich selbst mögen und du musst deinen Ort mögen, ihn erkennen, wertschätzen, es ist der Ort, an dem du lebst. Es ist also diese Beziehung zwischen Territorium, Raum, Selbstwertgefühl und Identität, die die Arbeit von Rosa hervorbrachte, all diese Informationen, eine Diskussion, die um diese Elemente organisiert wurde, und sogar mit dieser historischen Verbindung, ein weltweit anerkanntes Werk zu sein und dieses Gebiet hier zu einem großen Teil als seinen locus zu haben. Dann beginnt von da an diese objektivere und klarere Beziehung zwischen Rosas Werk und der Arbeit, die wir hier anregen. ${ }^{24}$

Beim Thema Selbstwertgefühl im Sertão, das in den Interviews oft erwähnt wurde, handelt es sich um einen wichtigen Punkt in den Leitlinien einiger der in der Region entwickelten Projekte. Die Faktoren, die als schwächend für ein solches Selbstwertgefühl wirken, sind unzählig, einschließlich der vorherrschenden und fehlgeleiteten Positionen und Vorstellungen, die oft von großen städtischen Zentren in Bezug auf ländliche Gebiete kommen. Um diesen problematischen Positionen zu begegnen, ist es wichtig, die Bedeutung der unterschiedlichsten Kulturen und Wissensformen zu erkennen und ihre Versuche der Hierarchisierung zu dekonstruieren, eine Position, die im Einklang mit Rosas literarischer Produktion steht.

24 Almir Paraka: wie Anm. 21. 


\section{Der Nachhall der sertanejo-Kultur: Rosianische Literatur als Kulturerbe und Impulsgeber für Initiativen sozialen Wandel}

Das von Rosas Literatur erzeugte «immaterielle Erbe〉 dient als Katalysator und Impulsgeber für verschiedene Initiativen und Projekte, die vor allem in den letzten zehn Jahren Früchte getragen haben. In diesem Zusammenhang werden wir ein Panorama erstellen, das darauf abzielt, einige Nachwirkungen des rosianischen Werkes in dieser Region des Sertão zu identifizieren und zu veranschaulichen. Die Zentren des Wandels, auf den laut der angesprochenen Richtlinien abgezielt wird, die um die Jahrtausendwende gestärkt und zusammengewachsen sind, stehen in direktem oder indirektem Zusammenhang mit ADISVRU («Agência de Desenvolvimento Integrado e Sustentável do Vale do Rio Urucuia», dt. "Agentur für Integrative und Nachhaltige Entwicklung des Rio Urucuia-Tals»). ${ }^{25}$ Nach José Idelbrando Ferreira de Souza ${ }^{26}$, einem der Gründer und Präsidenten von ADISVRU, hatte die Agentur zwei Hauptziele vor Augen: Konsolidierung und Diversifizierung der sozialen Basis sowie Diversifizierung der Produktionsbasis im gesamten Urucuia-Einzugsgebiet. ${ }^{27}$ Für die Schaffung selbsttragender Strukturen sowie

25 In seiner Definition handelt es sich um eine «Zivilgesellschaft mit nicht-wirtschaftlichen Zwecken, gegründet am 21. Dezember 2000, bezeichnet als OSCIP [Organização da Sociedade Civil de Interesse Público]. Ihre Aufgabe ist es, die menschliche und nachhaltige Entwicklung des Gebietes von Urucuia Grande Sertão durch die Steuerung des Wissens und die Anregung lokalen Engagements sowie die Diversifizierung der sozio-produktiven Basis und die Solidarwirtschaft zu fördern, mit dem Schwerpunkt auf der Verringerung von Armut und Ungleichheit.» Verfügbar unter https://www.umcanto.com/cities/arinos?locale=de. Letzter Zugriff: 31.1.2018 (unsere Übersetzung, Anm. d. Üb.).

26 Ferreira de Souza bestritt das Amt des Bürgermeisters von Arinos von 1997 bis 2000, dem Gründungsjahr von ADISVRU.

27 Wie Ferreira de Souza in einem am 24.1.2017 von mir in Arinos, Sertão von Minas Gerais, aufgezeichneten Interview schildert, agiert die ADISVRU «nicht nur in Arinos, sondern in der Region des Urucuia als Ganzes, ausgehend von dem Verständnis, dass keine Gemeinde sich entwickeln kann, wenn nicht auch die Region, in die sie eingefügt ist, sich entwickelt [...] Aus diesen Ideen heraus entwickelt das Projekt die interkommunalen Beziehungen. [...] Neben der interkommunalen Zusammenarbeit gibt es die Arbeit, die produktive Basis zu diversifizieren, [...] und die Arbeit, die soziale Basis zu diversifizieren, weil in der Vergangenheit lediglich die Bürgermeister und einige Ratsmitglieder die Akteure waren, die mit den Amtsträgern oder auch regionalen oder nationalen Politikern, seien es Abgeordnete, Staatssekretäre und Minister, sprachen. Mit der Diversifizierung der sozialen Basis wurden eine Diversifizierung der anderen Akteure, andere interkommunale und auch regionale Organisationen erarbeitet, und diese neuen gesellschaftlichen Akteure machen Veranstaltungen, Seminare bekannt, die Experten aus Bund und Landesregie- 
für die Autonomie und Entwicklung der gesamten Region waren Verbände mit lokalen Führungen von großer Bedeutung. Die Agentur spielt eine entscheidende Rolle bei der Verbindung von Projekten mit Fokus auf der Entwicklung der zwischenmenschlichen Interaktion und auf der Integration der lokalen Gemeinschaften, indem sie sie zu Protagonisten des regionalen Wandels machen, um ihnen bessere Lebensbedingungen, die Anerkennung des Wertes der lokalen Kultur und somit auch die Steigerung ihres Selbstwertgefühls und die Wertschätzung ihrer Identitäten zu ermöglichen. José Idelbrando weist darauf hin, dass für einen solchen Versuch

das Werk Grande sertão: veredas als Strategie zur Stärkung eines Gebietes der Identität gesucht wird, damit wir ausgehend von dieser Identität mit innovativen Projekten arbeiten können, die mit unserer Realität verknüpft sind, damit Entwicklung stattfindet. [...] Es werden gerade verschiedene Strategien entwickelt, um ausgehend von diesem Werk ein Gebiet der Identität zu konstruieren. ${ }^{28}$

In diesem Sinne, auf der Suche nach Vervielfältigung der sozialen Basis, findet sich unter den Projekten von ADISVRU die «Central Vereda» ${ }^{29}$, die verantwortlich

rungen anziehen, um an der Durchführung solcher Veranstaltungen mitzuwirken. So gab es einen größeren Austausch nicht nur mit Räten und Bürgermeistern, sondern auch mit diesen neuen, nach und nach auftauchenden Akteuren.»

28 Auszug aus dem Interview von Ferreira de Souza (wie Anm. 27).

29 «[M]it Sitz in der Stadt Arinos, setzt sich der Nordwesten von Minas Gerais aus 9 Produktionszentren zusammen, die auf die Gemeinden Natalândia, Sagarana/Arinos, Bonfinópolis de Minas, Riachinho, Serra das Araras/Chapada Gaúcha, Urucuia, Uruana de Minas, Buritis und Arinos verteilt sind. In einem Solidarischen Produktionsnetzwerk sind heute in dieser Region ungefähr 80 Handwerkerinnen und Handwerkern in der Initiative «Central Veredas» organisiert. Sie entstand durch neue Bemühungen mehrerer Partner, um Autonomie für die Legitimierung des Netzwerks als produktiver kultureller Revitalisierung zu schaffen, die mit Produktion und Einkommen gekoppelt ist, um ihre Zentren zu stärken. Dies gewährt den Teilnehmerinnen und Teilnehmern an der Initiative Zugang zum Markt, Qualifikationen, die Durchsetzung fairer Preise und die Verbreitung der handwerklichen Produkte, bei gleichzeitiger Ausführung sozioökonomischer und ökologischer Verteidigung, der Bekämpfung von Sklavenarbeit sowie der Verfechtung von Gendergleichheit. Die Organisation optimiert, orientiert und organisiert die gesamte Logistik und Komerzialisierung der von den Zentren produzierten Produkte in jeder Gemeinde, die zuvor isoliert arbeiteten. Derzeit produzieren die Zentren im Einklang mit ihrem Wesen, Frucht der Kreativität, Inspiration und Kultur des Urucuia-Tals. Es handelt sich um traditionelles Handwerk, dass heißt Techniken, die im Laufe der Jahre von Eltern an ihre Kinder weitergegeben wurden, mit Quailitätsbezug, Erfahrung, die von Generation zu Generation weitergegeben wurde. Alle Produkte werden auf ökologisch korrekte Weise produziert, wobei der Reichtum und die natürlichen und kulturellen Ressourcen der nordwestlichen Region von Minas Gerais, bekannt als Vale do Urucuia Grande Sertão Veredas, genutzt werden.» Verfügbar unter https://www.centralveredas.com.br/ sobre-nos. Letzter Zugriff: 31.1.2018 (unsere Übersetzung, Anm. d. Üb.). 
für die Bewahrung der Kultur der charakteristischen Sertanejo-Stickereien ist. Die Aktivität entspricht einer von alten Generationen herkommenden Tradition, welche durch den Fortschritt der Textilindustrie immer mehr an Raum verlor und um ein Haar im Begriff war, sich aufzulösen. Mit der betreffenden Initiative, die bei der Produktion und Kommerzialisierung behilflich war, wurde dieses kulturelle Merkmal in der Region wiederbelebt und so die Aufwertung handwerklicher Arbeit und eine Einkommen generierende Quelle ermöglicht.

In Bezug auf die Diversifizierung der Produktionsbasis war eine der Aktionen von ADISVRU die Schaffung der «Copabase Urucuia Grande Sertão» («Cooperativa Agroextrativista em Base da Agricultura Familiar Sustentável e Economia Solidária», dt. "Agrar-Extraktivistische Genossenschaft auf der Grundlage nachhaltiger Familienlandwirtschaft und Solidarwirtschaft») ${ }^{30}$, die, wie José Idelbrando in seinem Interview betont,

die Frage nach der Nutzung der Früchte des Cerrado einführend, den Extraktivismus konzipiert, um die Basis der familiären Landwirtschaft zu stärken und sich damit nicht nur die Genossenschaft positiv entwickelt, sondern auch die Genossen. Die Idee war, daran zu arbeiten, dass dem Produzenten außerhalb seiner Pforten eine bessere Einbindung und innerhalb seiner Pforten bessere Unterstützung ermöglicht wird, dass die Frage der Wertsteigerung ausgearbeitet wird und die Beschaffung von Produktionsmitteln sich verbessert, da oft teure Produktionsmittel gekauft werden und viele Hersteller Produkte gegen Produktionsmittel austauschen, wobei sie zuvor Verluste machten. ${ }^{31}$

Die Kooperation wurde 2008 anlässlich des hundertsten Geburtstages von João Guimarães Rosa ins Leben gerufen, wie von ihren Gründern ausgeführt wird. Sie wurde «mit starken Einfluss von Guimarães Rosas Denken gegründet und geografisch in einer Umgebung angesiedelt, die als Kulisse und Inspiration für die Produktion einiger seiner wichtigsten Werke diente». ${ }^{32}$

30 «Durch die Zusammenlegung von durchschnittlich 200 Familien der Familien-Landwirtschaft des Rio Urucuia-Tals ist es die Aufgabe der Genossenschaft, Arbeit und Einkommen für die Familien nach den Grundsätzen der Solidarischen Volkswirtschaft zu erwirtschaften. Gegründet am 23. Februar 2008, begannen ihre Aktivitäten im selben Jahr, die Genossenschaft ist in den Gemeinden Arinos, Buritis, Pintópolis, Formoso, Riachinho, Urucuia, Uruana de Minas, Bonfinópolis de Minas und Chapada Gaúcha tätig.» Verfügbar unter http://www.centraldocerrado.org.br/comunidades/copabase/. Letzter Zugriff: 31.1.2018 (unsere Übersetzung, Anm. d. Üb.).

31 Auszug aus dem Interview von Ferreira de Souza (wie Anm. 27). (Extraktivismus: gemeint ist eine nachhaltige und artenschonende Art der Bewirtschaftung naturnaher Landschaften, Anm. d. Üb.)

32 Verfügbar unter http://www.centraldocerrado.org.br/comunidades/copabase/. Letzter Zugriff: 31.1.2018 (unsere Übersetzung, Anm. d. Üb.). 
Angetrieben von diesem hundertjährigen Jubiläum, das für die brasilianische Kultur und insbesondere für die Region außerordentlich repräsentativ ist, begannen Ende 2007 in der Gemeinde Chapada Gaúcha die Aktivitäten des Instituto Rosa e Sertão, das von einer Gruppe von Pädagogen und Kulturakteuren gegründet wurde und mit der Mitwirkung der lokal Ansässigen rechnen konnte. Die Interessengemeinschaft entsteht mit dem Zweck der Aufwertung und Erhaltung der kulturellen und ökologischen Vielfalt der Region. ${ }^{33}$ Von Neuem zeigt sich die Verbindung zwischen einem solchen Projekt und der Vorstellungswelt des rosianischen Werkes, was sich in der Namenswahl des Instituts widerspiegelt. Damiana Campos, seine derzeitige leitende Koordinatorin, meint in einem Interview, dass sie, sogar bevor sie die Werke von Guimarães Rosa gelesen hatte, schon «alles über ihn» wusste:

ich hatte immer viele Geschichten gehört, denn es gab viele Geschichten über ihn, hier dreht sich alles um ihn: Es gibt einen Park namens Grande Sertão: Veredas, es gibt eine Straße namens Guimarães Rosa [...] Wie kann einer nicht alles über Guimarães Rosa wissen $?^{34}$

So wurde das erste Projekt des Instituts unter dem Titel Manuelzinho-da-crôa (dt. Der-kleine-Manuel-der-Sandbank) realisiert, dessen Name sich auf den schlichten Spruch der Figur Reinaldo zu Riobaldo im Roman von Rosa bezieht: «das niedlichste, reizendste Vögelchen flußauf- und abwärts [...], genannt Der-kleine-Manuel-der-Sandbank.» ${ }^{35}$ In dem Projekt bezog sich dieser schöne kleine Vogel auf die Kinder der Region, zu denen die pädagogischen und kulturellen Aktivitäten des Handwerks, des Geschichtenerzählens, der Musik, des Singens und des Tanzens zurückgekommen sind. Wie Damiana Campos bemerkt: «bewirkt die Arbeit mit den Liedern und der Musik, dass die Kinder das Unsere als von großer Wichtigkeit wertschätzen». So zielten etwa die Maculelê- und Capoeira-Tänze, die in dem Projekt gelehrt wurden, darauf ab, die afrobrasilianische und indigene Ge-

33 Damiana Campos, eine der Gründerinnen des Instituto Rosa e Sertão, kommentiert in einem Interview, dass die Anstalt «mit Fokus auf Aktivitäten mit regionalen Gemeinschaften gegründet ist, weil ihre Mitglieder die Erfahrung des ländlichen Raums bereits mitbringen. Basis von «Rosa e Sertão> war die Beteiligung von Lehrerinnen und Lehrern des öffentlichen Netzes, ländlichen Lehrern, Lehrern der Ausbildung auf dem Lande und von der Primärbildung. [...] Es ist eine Einrichtung mit femininer Basis, wo es mehrheitlich Frauen gibt».

34 Auszug aus dem Interview von Damiana Campos in der Gemeinde Chapada Gaúcha, Sertão von Minas, am 25.1.2017.

35 João Guimarães Rosa: Grande sertão: veredas. 20. Hg. Rio de Janeiro: Nova Fronteira 2001, S. 192. Vgl. Jõao Guimarães Rosa: Grande Sertão. Aus dem brasilianischen Portugiesisch von Curt Meyer-Clason. Köln: Kiepenheuer \& Witsch 1994, S. 135. 
schichte zusammen mit dem Geschichtenerzählen zu bewahren. ${ }^{36}$ Daiana Campos, auch Mitglied des Instituto Rosa e Sertão, erklärt, dass

\begin{abstract}
das Projekt «Manuelzinho-da-crôa» fast 200 Kinder zusammen führte, die mit Musik arbeiten, und mit der Musik haben wir ein tolles Resultat erzielt, mit den Auszügen aus Guimarães Rosa, wo man Kinder von fünf oder acht Jahren sah, die Grande Sertão lasen. Wir hatten das Vergnügen, das hundertjährige Jubiläum von Guimarães mit diesen Kindern zu feiern, die die Arbeit von Guimarães Rosa lesen, und für uns ist es eine große Befriedigung, hier in unserer Gemeinde einen Raum zu haben, der die Volkskultur fördert und und die Frage nach der Impulsgebung zur Lektüre vorantreibt. ${ }^{37}$
\end{abstract}

In Bezug auf Projekte des Instituts kommentiert Damiana Campos: «[...] Mit Poesie haben wir es geschafft zu kämpfen, die Poetik, das Wort war die Wahl des Instituto Rosa e Sertão». ${ }^{38}$ Indem sie nochmals die Intertextualität des rosianischen Werkes im Namen der Musikgruppe «Diadorinas» hervorhebt, die von den Campos-Schwestern gegründet wurde, ${ }^{39}$ antwortet Damiana auf die Frage nach dem Einfluss, den die rosianische Literatur in der Region ausübt:

Ich denke, dass unsere Projekte, alles, was wir tun, viel von ihm hat, selbst wenn es verleugnet wird. Wir leugneten das viele Jahre, «Ach wie ätzend, ständig muss von Guimarães Rosa die Rede sein». Doch selbst das Leugnen passierte, weil wir so sehr über ihn sprechen wollten. [...] Guimarães ist da, er ist unsterblich geworden, und wenn er unsterblich wird, soll das heißen, dass er vorübergehen kann, dass er dies bereits ist. Man kann alle Bücher verbrennen, denn er ist schon in die mündliche Tradition übergegangen. Du kannst das ganze Volk fragen, wie die Geschichte des Grande Sertão lautet, alle Welt weiß es, nicht alle Welt, aber zumindest, wer Teil des Nukleus ist. ${ }^{40}$

Immer noch von den Feierlichkeiten zum 100. Geburtstag von Guimarães Rosa angetrieben wurde 2008 das Sagarana Festival geschaffen, das bereits zum siebten Mal stattfindet und das unter anderem die Aufwertung des Selbstwertgefühls der Gemeinden im Urucuia-Tal zum Ziel hat, die Aufwertung der kulturellen sertane-

36 Siehe dazu: https://rosaesertao.org.br.

37 Auszug aus dem Interview von Damiana Campos, durchgeführt in der Gemeinde Chapada Gaúcha, Sertão von Minas, am 25.1.2017.

38 Auszug aus dem Interview von Daiana Campos (wie Anm. 34).

39 Mit Damiana, Daiana und Diana, den Campos Schwestern, alle drei an der Verwaltung des Instituto Rosa e Sertão tätig, wurden Interviews im «Ponto de Cultura Seu Duchim dos Gerais Espaço Geral de Folias» (dt. «Kulturpunkt Seu Duchim dos Gerais - Allgemeiner Ort für Festlichkeiten») durchgeführt. Die Einrichtung, die fürs Angebot eines kulturellen Programms fürs regionale Publikum verantwortlich ist, dient derzeit als Hauptsitz des Instituts.

40 Ebda. 
jo-Identität, sowie Diskussionen über die solidarische, nachhaltige Entwicklung, den agrarökologischen Übergang und die Erhaltung der Wasserressourcen. ${ }^{41}$ Das Problem des mangelnden Umweltbewusstseins und der durch menschliches Handeln verursachten Umweltauswirkungen wird zum Beispiel im Text Uma estória de amor (dt. Eine Liebesgeschichte) herausgearbeitet. Wie in den Richtlinien des Festivals beschrieben, verspricht sein abwechslungsreiches Programm «Volkskultur, traditionelles Wissen und Praktiken des mineirischen Sertão sowie soziale Technologien, die sowohl die ländlichen Gemeinden und die Städte des Rio Urucuia Tals als auch Menschen und Gruppen aus Minas Gerais, Distrito Federal, São Paulo und anderen Staaten zusammenführen».42

Das Festival, das 2010 zum dritten Mal stattfand, ermöglichte die Einweihung eines weiteren wichtigen Akteurs für die soziale und kulturelle Entwicklung der Region, des Instituto CreSertão («Centro de Referência em Tecnologias Sociais do Sertão», dt. «Referenzzentrum für soziale Technologien des Sertão»). Es ist ein gutes Beispiel für den Nachhall der Projekte, die ausgehend vom Fluss Urucuia und der Arbeit von Guimarães Rosa zu einem Netz von Dialogen führen, welche den Wandel und die öko-soziokulturelle Entwicklung der Region intensivieren. Silva weist darauf hin, dass «im Projekt die Begründung des Namens des Instituts CreSertão den Wert von JGR als inspirierende Quelle darstellt und die These bekräftigt, dass der Autor im urucuianischen Raum eine herausragende Persönlichkeit ist und eine wichtige Rolle bei der Gruppenbildung spielt.» ${ }^{43}$ Das CreSertão-Institut, das als Nichtstaatliches Projekt mit Sitz in Sagarana fungiert, übt seine Tätigkeit in einem vom Staatlichen Forstinstitut von Minas Gerais («Instituto Estadual de Florestas de Minas Gerais», IEF-MG) gewährten Gebiet aus. Es «arbeitet daran,

41 Almir Paraka hebt in seinem Interview (wie Anm. 27) die Agrarindustrie hervor: «die Agrarindustrie, die Spitze der Modernisierung und Beschäftigung des brasilianischen Cerrado, die heute auch diesen Durst nach Wasser hat, durch Bewässerungssysteme, die eine absurde Macht darstellen, die analysiert, überprüft und kontrolliert werden muss. Eine grundlegende Ressource wie Wasser, die als reine Ware behandelt wird, und zwar auf dem Schwarzmarkt, außerhalb des gesamten Sektors der Regulierung und Standardisierung der Zulassung von Wasser in Brasilien, das sind Themen, die sich überschneiden, heute bin ich in einer Militanz».

42 «Während des Festivals verwandelt sich Sagarana in ein lebendiges Zentrum des Austauschs und der Erfahrungen, indem es verschiedene Generationen und soziale Kontexte integriert: Jung und Alt; städtische Bevölkerung; Forscher, Studenten und Meister der mündlichen Überlieferung; Spinnerinnen, Folias de Reis, Musikgruppen und zeitgenössische Percussion-Gruppen; Familienbauern und Lehrlinge unter anderem der Agrarökologie und Permakultur. All diese Menschen treffen sich und finden sich in kulturellen Darbietungen, Workshops und Dialogen, Food Courts mit typischen Lebensmitteln, ökologischen Spaziergängen und spontanen Treffen wieder». Verfügbar unter https://festivalsagarana.wordpress.com/o-festival/. Letzter Zugriff: 30.1 .2018 (unsere Übersetzung, Anm. d. Üb.).

43 Silva: Nesta água que não para, S. 47. 
das Ethos des Sertão zu fördern, um diejenigen zu qualifizieren, die in der Region leben [...] und präsentiert Vorschläge für Nachhaltigkeit in Bezug auf die Umwelt.» ${ }^{44}$ Bei den entwickelten Maßnahmen handelt es sich um Strategien zur Generierung von Einkommensquellen und zur Verbesserung der sozialen Bedingungen in den Gemeinden. Unter seinen Tätigkeiten heben sich die «Sozialen Technologien, soziale Mobilisierung, ökologische Kompetenz, Ausbildungs- und Qualifizierungsexperten, Projekte zur Schaffung von Arbeit und Einkommen, Umweltschutz und Stärkung der kulturellen Identität des Sertão von Minas Gerais» ${ }^{45}$ ab. Es ist auch eines der Projekte der «Universidade das Culturas» (UniCult, dt. «Universität der Kulturen»). ${ }^{46}$

Auf diese Weise macht sich die Präsenz des immateriellen Erbes der rosianischen Literatur als Anreiz und Potenzial für die soziale und kulturelle Entwicklung der Region bemerkbar, basierend auf verschiedenen Projekten und Initiativen, die miteinander in Beziehung stehen, sich gegenseitig beeinflussen und auf eine gemeinsame Art und Weise ein immer stärker zusammenhängendes und repräsentatives Netzwerk bilden. Das Bild der letzten Ausgabe des Sagarana Festivals, das 2015 stattfand, ist ein interessantes Beispiel für die Wahrnehmung dieses Aspekts.

\section{Ebda.}

45 Verfügbar unter http://unicult.org/project/cresertao/. Letzter Zugriff: 31.1.2018 (unsere Übersetzung, Anm. d. Üb.)

46 «Die Universität der Kulturen ist eine Reihe von Initiativen, die Forscher, Gruppen, Kollektive, Universitäten, Kulturzentren und kulturelle Akteure verbindet. Ziel ist es, Projekte im Bereich der kulturellen Bildung zu vernetzen. Zu diesem Zweck basiert UniCult auf der Erkenntnis, dass es mehrere legitime, autonome und kulturell anerkannte, empirische und theoretische, angestammte und zeitgenössische, städtische und ländliche Ausbildungsmethoden gibt, die über eine traditionelle Konzeption der Schule hinausgehen, die auf Beschränkung und Fragmentierung von Wissen beruht. Mit den neuen Ressourcen, die im 21. Jahrhundert verfügbar sind, schafft das Netzwerk Räume für die Konvergenz von Institutionen und kulturellen Akteuren, verbindet die verschiedenen traditionellen oder innovativen Trainingsmethoden und schafft Debattierraum für Konzepte, Werte und Ideen, die momentan noch in starren Institutionen und akademischen Modellen zentriert sind.» Verfügbar unter http://unicult.org/\#sobre. Letzter Zugriff: 31.1.2018 (unsere Übersetzung, Anm. d. Üb.). 


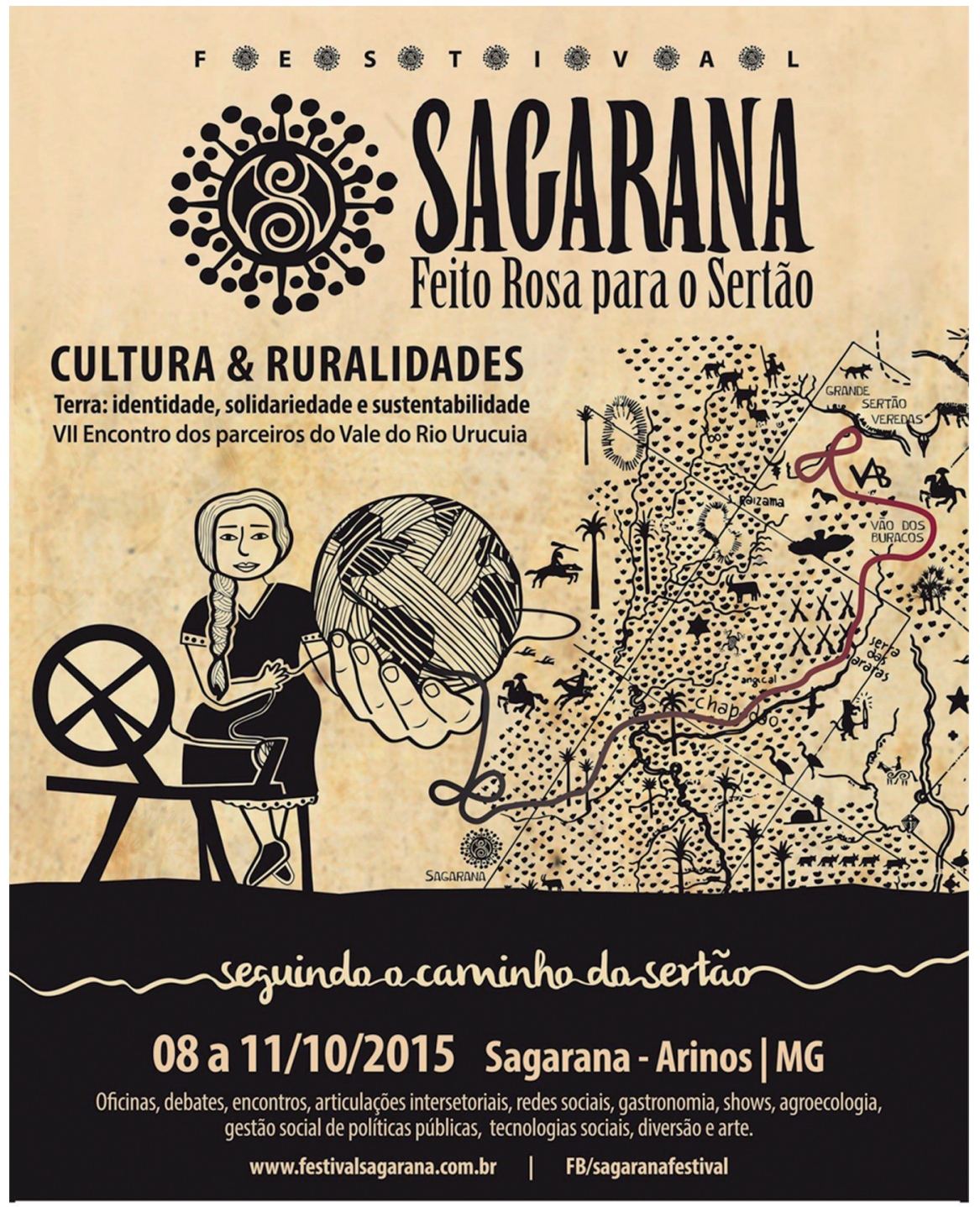

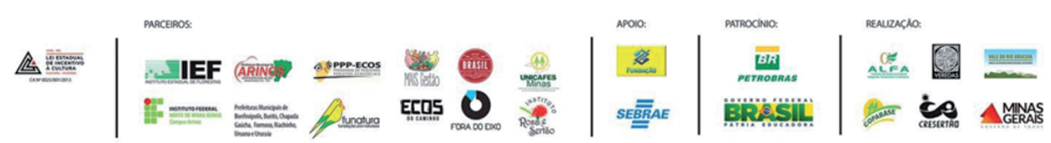

Abb. 1: Sagarana Festival, 2015. Bild entwickelt von der Grafikdesignerin Amanda Rabelo Cardoso 
Das Bild der Spinnerin, die den Faden spinnt, der, anfänglich schwach, zu einem großen, als Globus gestalteten Ball wird, ist äußerst repräsentativ, da er symbolisch für viele der Früchte steht, die in den letzten Jahren bereits geerntet wurden, dank der von vielen Personen mit Hingabe und Ernsthaftigkeit entwickelten Arbeit sowie der in die verschiedenen erwähnten Projekte involvierten regionalen Institutionen. Es zeigt auch das Potenzial auf, das für die Ausarbeitung der verschiedensten Arten der bordados (dt. Stickereien) generiert wird. In der Illustration hat der Faden, aus dem das Knäuel besteht, zwei Knoten, die durch das Symbol des Unendlichen repräsentiert werden, unter direktem Verweis aufs mathematische Unendlichkeitszeichen, das den Roman Grande sertão: veredas abschließt. Das zusammengesetzte Bild beruht auf der Reproduktion der Illustration, die der Künstler aus Paraná Poty Lazzarotto für die Darstellung des rosianischen Sertão in der ersten Ausgabe des Romans entwickelt hat. Auf diesem entfaltet sich der Faden des Knäuels, der durch die zwei Knoten/Unendlichkeitssymbole gekennzeichnet ist, die zwei sehr relevante Punkte zu erkennen geben: Sagarana und Grande sertão: veredas, Ausgangs- und Wendepunkt der literarischen Laufbahn des Schriftstellers, sowie Ausgangs- und Wendepunkt der von den Wanderern des Projektes «O Caminho do Sertão» zurückgelegten Durchquerung.

Hinsichtlich des Einflusses der rosianischen Gedanken und der Projekte in der Region, die auf seinem Werk basieren, kommentierte Agemiro Graciano de Jesus, ein Einwohner, ehemaliger Viehtreiber, Bauer und Wegführer im Projekt «O Caminho do Sertão»: «die Bewegung Guimarães Rosa hier in Sagarana war ein Anfang, es war ein Sprung, denn da kam das Fest Guimarães Rosa, es kam der Weg [des Sertão].» ${ }^{47}$ Über dieses Projekt sagt José Idelbrando, dass es «vom Jahr 2000 an entwickelt wurde, als Almir Paraka Bürgermeister von Paracatú war und ich Bürgermeister von Arinos. Von da an träumten wir von dieser integrativen und nachhaltigen Entwicklung im Flussgebiet von Urucuia.» ${ }^{48}$ Die Gründung des Projekts gehört der gleichen Epoche an wie die Entstehung der ADISVRU des Urucuia-Flusstals, Hauptverantwortliche für die Ermöglichung eines funktionierenden Netzwerks der genannten lokalen Initiativen und Projekte. Folglich werden, koordiniert von ADISVRU, ab 2013 die innovativsten Projekte der Agentur initiiert, die laut José Idelbrando in vier Achsen organisiert sind:

kulturelles Gedächtnis, soziale Innovation, kreative Ökonomie, oder wie einige es nennen, Kulturwirtschaft, und Nachhaltigkeit. Innerhalb jeder dieser Achsen werden die spezifischen, einzigartigen und innovativen Projekte ausgearbeitet. Der «Caminho do Sertão» ist

47 Auszug aus dem Interview von Agemiro Graciano de Jesus in Sagarana am 23.1.2017.

48 Auszug aus dem Interview mit Ferreira de Souza (wie Anm. 27). 
in diesem Vorschlag enthalten, weil er Anlass gibt, über die Frage der Erinnerung und sozialen Innovation nachzudenken. ${ }^{49}$

Auf diese Weise ist «Caminho do Sertão» ein heute für die betreffenden Diskussionen sehr repräsentatives Projekt. Es stellt für den fruchtbaren solidarischen Vorschlag ein weiteres gutes Beispiel dar, da es auf die Arbeit zahlreicher Kulturproduzenten, regionaler Führer, Anwohner sowie auf den Beitrag verschiedener Institute und Partnereinrichtungen ${ }^{50}$ zählen kann. Zählen kann es ebenso auf die Arbeit der lokalen Kommunen entlang der gesamten Strecke, die laut Diana Campos «die Grundlage von allem bilden, der gesamten Bewegung und Produktion, die den «Caminho〉 ausmacht». ${ }^{51}$ Wie in seinen Richtlinien dargelegt wird, beabsichtigt der Weg «das Eintauchen in Guimarães Rosas Universum, in die Literatur, in die Geographie, in das Wissen und die Praktiken der Bewohner der Flusstäler Urucuia und Carinhanha im Nordwesten und Norden von Minas Gerais».52

49 Wie José Idelbrando im Interview (wie Anm. 27) erklärt, «lautet die Frage auf dieser Achse des sozialen Gedächtnisses: Woher kommen wir? Wo sind wir? Wohin gehen wir? Wie geht es uns? Fast alle Mechanismen, Strategien sind also auf diese retrospektive und prospektive Erinnerung ausgerichtet. [...] Die andere Achse, also die der sozialen Innovation, wenn wir in der Vergangenheit der sozialen Frage nach einer eher paternalistischen, manchmal sogar klientelistischen, manchmal auch ein wenig demagogischen Tendenz nachgehen, ist dieses neue «sozial», dass man diskutieren möchte, das «Erwache!» der Potenzialisierung dieser sozialen Akteure, seit die Flussanliegerbevölkerung über Alternativen für Nachhaltigkeit nachdenkt.»

50 Daten über die 1. Ausgabe des Projekts - Umsetzung: Vale do Urucuia (ADISVRU). Partner: Copabase, Instituto Rosa e Sertão, CreSertão, Funatura, Instituto Federal do Norte de Minas (Campus Arinos, dt. «Bundesinstitut für Nord-Minas`), Instituto Estadual de Florestas (IEF-MG, dt. «Staatliches Forstinstitut), Unipaz-DF, Fora do Eixo, Prefeitura Municipal de Arinos-MG (dt. «Gemeindeverwaltung von Arinos-MG), Prefeitura Municipal de Chapada Gaúcha-MG (dt. «Gemeindeverwaltung von Chapada Gaúcha-MG»). Unterstützung: Secretaria de Cultura do Governo de Minas Gerais (dt. «Kulturabteilung der Regierung von Minas Gerais`).

51 Auszug aus dem Interview von Diana Campos, in der Gemeinde Chapada Gaúcha, Sertão von Minas, am 25.1.2017.

52 «So führt uns eine literarische Reise «von Sagarana zum großen Sertão〉 vom ersten bis zum wichtigsten Werk Rosas. Es wird eine Reise vorgeschlagen, die einen Teil des Weges abdecken wird, den Riobaldo bei seiner Durchquerung in Richtung der Ebene der Sussuarão - der vermeintlichen Wüste von Grande sertão: veredas; eine Reise in Gebiete, die durch Bewegungen, Verschiebungen und Wendungen gekennzeichnet sind, durch Begegnungen auf Kreuzwegen, um zu zeigen, dass die Wüste nicht nur Wüste ist, sondern Land eines «geraizeiro» Volkes, in dem Natur und Menschlichkeit miteinander verwoben sind, Kulturland! Es ist ein sozio-ökologischer Weg für die Vielfalt des Cerrado von Minas Gerais, in dem Querungen, Lagunen, Flüsse, traditionelle Kommunen, Siedlungen, Siedlungen der Agrarreform und große Fazendas der Agrarindustrie verschmelzen. Es ist eine Gelegenheit für Wanderer, auf den Wüstenbildungsprozess zu schauen, der die Region bedroht und über die notwendigen Veränderungen nachzudenken, um ihn zu vermei- 
De facto ist, eingetaucht in sehr ähnliche Umgebungen, die den fiktionalen Texten von Guimarães Rosa als Triebkraft dienten, so wie dem Charakter Grivo oder sogar dem Schriftsteller selbst auf seinen Reisen, die Faszination oft unvermeidlich. Manchmal ist es möglich, sich vorzustellen, eine Figur in Rosas Geschichten zu sein und auf dem Weg auf Menschen zu stoßen, die als Miguilins, Ditos, PêBois, Rosalinas, Manuelzões, Glorinhas, Camilos, Doraldas oder Caras-de-Bronze identifiziert werden könnten.

Während des Spaziergangs wird man dazu gebracht, die natürliche Schönheit der Cerrado-Regionen mit ihrer Flora und Fauna zu erleben, die im Kontrast zu den monokulturellen Latifundien mit Sojabohnen oder Grasplantagen steht, was hauptsächlich im Umland der Gemeinde Chapada Gaúcha stattfindet. Die Erfahrung des Durchquerens ist auch in die Klarheit der agrarökologischen Vorstellung vieler Erzeuger landwirtschaftlicher Familienbetriebe in der Region gehüllt, was sowohl auf die täglichen Erfahrungen als auch auf die Kurse zurückzuführen ist, die von Initiativen im Zusammenhang mit diesen Projekten angeboten werden. Ein weiterer sehr interessanter Aspekt der Wanderung ist der ständige Kontakt und das Erlernen von Volksweisheiten durch die Führer und Einheimischen. ${ }^{53}$ Über den Gehalt dieser Art von Erfahrung spinnt seo Agemiro im Interview mit all seiner Einfachheit und großen Weisheit den folgenden Kommentar: «Du hast den Weg gemacht, der Weg muss erlebt werden, erst indem du den Weg gehst, weißt du, was der Weg ist.» ${ }^{54}$ Diese Reflexion macht die Notwendigkeit der Durchführung einer solchen Erfahrung als Brauch deutlich, um auf diese Weise ein umfassendes Verständnis für das zu erreichen, was sie bietet. Dieser Kommentar erinnert uns an die interessante Überlegung, dass «das Reale nicht in der Abreise oder der Ankunft liegt: es liegt für uns mitten in der Durchquerung.» ${ }^{55}$

den». Verfügbar unter https://ocaminhodosertao.wordpress.com/2014/05/13/o-caminho-do-sertao-de-sagarana-ao-grande-serta-veredas-2/. Letzter Zugriff: 30.1.2018 (unsere Übersetzung, Anm. d. Üb.).

53 Dieser Aspekt ist einer der entscheidenden Punkte des Projekts und laut Ferreira de Souza (wie Anm. 21), «ist die Erwartung, dass dieser Kontakt neben mehreren innovativen Möglichkeiten einen Austausch hervorbringt, und dass die Menschen, die hierher kommen, wissenschaftliche Kenntnisse mitbringen können oder auch anderes Wissen über andere Realitäten, was eine Suche und ein Austausch mit Volksweisheiten, dem Wissen der Bewohner des Flussgebietes, dem Wissen der arbeitsamen Landarbeiter ist, dieser Austausch kann ein Wissen generieren, das sehr relevant für unsere lokale Realität ist».

54 Auszug aus dem Interview von Agemiro Graciano de Jesus (wie Anm. 47) (seo: Redensart, respektvolle Anrede, Abk. senhor, die in der mündlichen Sprache weit verbreitet ist und in der Literatur von Guimarães Rosa auch viel verwendet wird, Anm. d. Üb.).

55 Rosa: Grande Sertão, S. 97. 
In Bezug auf die Wirkung des Projekts als einer weiteren wichtigen entwickelten Strategie auf der Basis des von Rosas Literatur generierten Potenzials mit der Absicht, den ersehnten Veränderungen in der Region Kontinuität zu verleihen, kommentiert Almir Paraka:

die ganze Verbundenheit mit Rosa kam zustande und von da an begann er, die Handlungen stark zu orientieren/zu beeinflussen. Der Versuch der Aktivitäten hier, des «Caminho do Sertão», ist also ein Versuch, die Fragen, die Rosa immer gestellt hat, in die Gegenwart zu überführen und in Taten und Erfahrungen umzusetzen. Vor allem in Erfahrungen. Die Menschen können viele Problematiken erfahren, von denen Rosa berichtet. Er arbeitet sie in seinen Erzählungen aus, er wollte ja immer zu Reflexionen anregen, zum möglichen Überdenken des Denkens. Der «Caminho do Sertão» ist also ein Stück weit ein Versuch, all diese Fragen, ihre Kreuzungen und ihre Einbeziehung auf die Gegenwart, die Aktualität, anzuwenden, um ein Feld der Reflexion zu schaffen, das dieser Region unter anderem auch helfen kann, die Möglichkeiten für seine Bewohner zu erweitern, was für viele Auswärtige, die hier durchreisen wollen, nützlich sein kann, zum Beispiel Stadtmenschen, die in großen Zentren leben und die Lebensweise der Menschen auf dem Land und die Werte dieser Menschen nicht kennen. ${ }^{56}$

Das Projekt hat bedeutende Fortschritte in diese Richtung gemacht, und tatsächlich beeinflusst die Intensität der Erlebnisse vor Ort die Wahrnehmung von Kultur und sozialer Dynamik, insbesondere im Sertão, aber auch in der Gesellschaft Allgemeinen.

Der Ankunftsort der Durchquerung des Sertão ist die Gemeinde Chapada Gaúcha, zentrale städtische Niederlassung des Nationalparks Grande Sertão Veredas. ${ }^{57}$ Das Projekt «O Caminho do Sertão» fungiert an dieser Stelle als eine Art Nebenströmung des traditionellen «Treffens der Völker von Grande Sertão Veredas», das jährlich im Juli und bereits zum 16 . Mal stattfindet. ${ }^{58}$ Wie üblich bietet

56 Auszug aus dem Interview von Almir Paraka (wie Anm. 27).

57 «Der Nationalpark Grande Sertão Veredas befindet sich an der Grenze der Bundesstaaten Minas Gerais und Bahia, mit Hauptsitz in der Gemeinde Chapada Gaúcha. Er hat eine Fläche von 230.671 Ha. Der Umfang des Parks beträgt 282.341.956 Meter. [...] Das Gebiet des Parks umfasst zwei Bundesstaaten und gehört zu den brasilianischen Naturschutzgebieten. Die 8.875 Hektar, die sich im Norden der Gemeinde Arinos befinden, beeinflussen die Beziehungen zwischen dem Sertanejo und der Natur und zwischen dem Sertanejo und seiner Kultur» (Silva: Nesta água que não para, S. 55). Der Forscher weist darauf hin, dass der Nationalpark «Grande Sertão Veredas» «die größte Attraktion des Kreises Urucuia Grande Sertão ist [...] [dieser], der sich durch mehrere Gemeinden mit Bezug zum Werk von JGR erstreckt. Darüber hinaus bringen die Wörter Grande und Sertão in der Bezeichnung fraglos den Kreis dem Werk von JGR näher» (Silva: Nesta água que não para, S. 54). (Unsere Übersetzung, Anm. d. Üb.).

58 Diese letzte Ausgabe wurde erneut von ADISC (Agência de Desenvolvimento Local, Integrado e Sustentável de Chapada Gaúcha, dt. Agentur für Lokale, Integrierte und Nachhaltige Entwick- 
die Veranstaltung Einheimischen und Besuchern verschiedene kulturelle und ökologische Attraktionen mit typischen Speisen, Shows, Poesieabende, Kunsthandwerk, Kunstausstellungen und verschiedene runde Tische zu Familienlandwirtschaft, Bildung auf dem Land, Umweltbewusstseinsbildung und Tourismus in der Region der Völker des Grande Sertão Veredas, die große Anziehungskraft hat und Menschen aus dem ganzen Land aufgrund der in der Region präsenten rosianischen Vorstellungswelt anzieht. Die Ankunft der Wanderer und die Mitwirkung beim Festival stellt eine weitere Attraktion dar und repräsentiert die Integration der Völker und kulturellen Projekte der Region des Urucuia-Beckens. Zu den Ergebnissen solcher Projekte in der Region behauptet Silva:

Die Anwesenheit von JGR nimmt Form an in kulturellen Initiativen, die darauf abzielen, das dem Sertão Eigene, seine Linien und Nuancen bis hin zur Errichtung der Identität des Urucuiano aufzuwerten. Die menschlichen Akteure, die in den Institutionen agieren, bilden kleine Knotenpunkte des großen Netzwerks, das sich im Tal der Urucuia ausdehnt. ${ }^{59}$

Die Durchführung des Projekts «O Caminho do Sertão», als spezifisches Beispiel für den Nachhall der sertanejo-Kultur, welcher der Literatur von Guimarães Rosa zu verdanken ist und der an seinem Ursprungsort nachklingt, fungiert als Tor zum Sertão und ist hinsichtlich seiner Dynamik sowohl für allgemein Interessierte als auch an Forscher in bestimmten Bereichen gedacht. Laut José Idelbrando Ferreira de Souza ist das Projekt seit seiner Entstehung «an Menschen gerichtet, die daran interessiert sind, die von Guimarães Rosa insbesondere in Grande sertão: veredas und auch in Sagarana so treffend beschriebene Kultur des Sertão von nahem kennenzulernen.» ${ }^{60}$ So ermöglichen seine Ausgaben Entdeckungen, die informell wie formell zur Stärkung anderer lokaler Projekte beitragen, mit dem Aufkommen neuer kultureller Initiativen in der Region ${ }^{61}$ sowie in Fragen, die sowohl die Kul-

lung von Chapada Gaúcha) durchgeführt, die als einen ihrer Unterstützer das «Instituto Rosa e Sertão» hat. Verfügbar unter http://portalveredas.com.br/2017/08/19/xvi-encontro-dos-povosdo-grande-serta-veredas/. Letzter Zugriff: 30.1.2018.

59 Silva: Nesta água que não para, S. 45.

60 Auszug aus dem Interview von Ferreira de Souza (wie Anm. 27).

61 Wir heben besonders das «Ecos do Caminho Sertão» («Echos des Weges des Sertão») hervor, «einer Initiative von Wanderern, die an der ersten Ausgabe des Projekts teilgenommen haben, unter Einbindung von Pädagogen, Künstlern, Studenten, Selbstständigen und Forschern in Verbindung mit Kultur und Nachhaltigkeit». Verfügbar unter https://www.catarse.me/ecosdocaminho. Letzter Zugriff: 30.1.2018 (unsere Übersetzung, Anm. d. Üb.). Als Frucht der ersten Ausgabe des Projekts «O Caminho des Sertão», integrierten sie die Gruppe der Organisatoren in die 2. Ausgabe desselben. Zusammen mit dem "Instituto Rosa e Sertão" organisierten sie im Oktober 2017 die erste Ausgabe der «CineBaru - Mostra Sagarana de Cinema» («Sagarana-Kinofilmvorführung») im Dorf Sagarana. 
tur des Sertão als auch die rosianische Literatur in Brasilien ${ }^{62}$ und sogar im Ausland ${ }^{63}$ betreffen.

Der repräsentative Einfluss von Rosas Werk, der im Raum des Sertão durch Projekte dieser Art deutlich wird, hat verschiedene Auswirkungen und trägt zur Entwicklung von Initiativen bei, die die soziokulturelle Dynamik der Region verändern. Wie José Idelbrando hinsichtlich der rosianischen Literatur als Grundlage von Initiativen sozialen Wandels hervorhebt,

nutzen wir diese als Anker. Sie ist immer der Anker, weil es eine Frage der größeren Identität ist. [...] Ausgehend vom [rosianischen] Werk geht es um die Stärkung eines Territoriums, von Wissen, Wirtschaft, Kultur, um den Erhalt unseres Wissens und unserer Volkskulturen. So ermöglicht uns das Werk, auf verschiedenen Wissensgebieten zu arbeiten. ${ }^{64}$

Jede Ausgabe dieser Projekte verstärkt die Förderung von Reflexionen über die sertanejo-Kultur, die Umwelt, das Werk von Guimarães Rosa, sowie den Zugang zu literarischer Kultur, die Aufwertung und Bewahrung nicht-literarischer Kultur, die Erhöhung des Selbstwertgefühls, die Anerkennung des Werts der Volksweisheit, sowie die Ermöglichung von sozioökonomischen Verbesserungen. In Bezug auf einige der Veränderungen in den Standpunkten, die von den lokalen Einwohnern als Folge der Realisierung solcher Initiativen in der Region wahrgenommen werden, hebt Almir Paraka hervor, dass

die Erfahrung, die wir zu machen beginnen, ist, dass sie [die einheimische Bevölkerung] sich selbst in der Arbeit entdeckt und wiedererkennt, dass das, was das Werk sagt, sie selbst betrifft, ihre Lebensweise, ob jetzt oder in jüngerer Vergangenheit, die Zeit ihrer Eltern und

62 Als Beispiel zietiere ich den mündlichen Vortrag: Todo abismo é navegável a barquinhos de papel: atravessando o sertão a pé, (dt. Jeder Abgrund ist mit Papierbooten befahrbar: zu Fuß durch den Sertão), das ich auf dem Kolloquium Outras Margens - 50 anos sem Guimarães Rosa (dt. andere Ufer - 50 Jahre ohne Guimarães Rosa), präsentierte, welches im August 2017 an der Universidade Federal do Paraná (UFPR-Curitiba) stattfand, als Ergebnis der Erfahrungen während der 3. Auflage des Projekts «O Caminho do Sertão» im Jahre 2016. In diesem Sinne wurde der Dokumentarfilm Sertanias mit Drehbuch und Regie von Alexandre Roldão und Juliana Dametto Guimarães Rosa während der 4. Edition des Projekts «O Caminho do Sertão» im Jahr 2017 gedreht und im selben Jahr landesweit ausgestrahlt. Verfügbar unter https://globosatplay.globo.com/globonews/v/6304800/. Letzter Zugriff: 30.1.2018.

63 Als Beispiel nenne ich aus der mündlichen Vortrag Refazendo a pé a travessia do sertão de Riobaldo (dt. Zu Fuß auf den Spuren von Riobaldos Durchquerung des Sertão), präsentiert von Willi Bolle auf dem Simpósio Guimarães Rosa e Meyer-Clason - literatura, democracia, saber-conviver (dt. Symposium Guimarães Rosa und Meyer-Clason - Literatur, Demokratie, Zusammenleben-Wissen), was im November 2017 im Iberoamerikanischen Institut (IAI-Berlin) gehalten wurde, als Frucht der Erfahrungen des Forschers in der 4. Ausgabe des Projekts «O Caminho do Sertão» 2017. 64 Auszug aus dem Interview von José Idelbrando (wie Anm. 27). 
Großeltern, viel von dem, was noch da und hier in der Region sehr lebendig ist, all das eröffnet Horizonte, eröffnet Perspektiven. ${ }^{65}$

Diese Eigenschaften werden im Interview mit Fidel Carneiro Araújo, einem anderen lokalen Einwohner, Erzieher, Landwirt und Führer des Projekts «O Caminho do Sertão» deutlich. In seinen Aussagen kann man feststellen, dass sich die Perspektive bezüglich der Art der Lektüre und der Identifikation mit der Kultur und der lokalen Identität, die von solchen Initiativen eröffnet werden, ändert:

Ich habe einen Kurs als ökologischer Führer gemacht und bin so in diesen Bereich gekommen [...] Das war es, was mich bewegte, denn ich habe meinen Heimatort immer mit ein wenig Geringschätzung betrachtet, alle hier dachten so. Es gab die traditionellen Arbeitsweisen, die Dinge hier zu tun, aber man hat dem nie einen großen Wert zugesprochen, bis ich gesehen habe, dass das alles ein Riesenpotenzial hat, dieser Kurs hat mir die Augen für das geöffnet, was ich in meinem Garten habe, was mein Nachbar dort hat, für die Expertise, die die Ältesten hier haben und der Kurs war es, der mich motivierte, diesen Kreislauf zu bewahren. [...] Mir wurde das Werk Grande sertão: veredas vorgestellt, das mich anfangs wegen seines großen Umfangs erschreckte, «Ob mich das wirklich so sehr reizen wird?», aber dann schien es so, als würde ich mit dem Typen selbst hier herumlaufen, und er selbst am reden. Sogar einige Redensarten meines Großvaters, total veraltet, komische Wörter: «Aber Großvater, was ist das denn?» Das kam vor, es taucht ab und zu auf. Die Jagunço-Geschichten, die mein Großvater erzählte, schilderte er genau so, dass die Leute aus dem Haus liefen: «Schau nur, sie kommen, die Rebellen», alleman verschwand aus dem Haus, sie ließen nur das Essen, das Zeugs dort, sie kamen, stopften sich die Bäuche voll, manchmal war es ihnen auch egal, ein paar fiese Kerle schikanierten die Leute, andere weniger, aber die Geschichten stimmten mit dem Buch überein, es kommunizierte viel mit mir. Und als mir das klar wurde, war das Buch schon zu Ende, es endete und begann gleichzeitig, weil es aufhört und du es direkt wieder von Anfang lesen willst, um die ein oder andere Sache wieder ins Gedächtnis zurückzurufen, also es ist ein Buch, dass du auf jeden Fall lesen, wieder lesen und leben wirst und es hat de Facto noch viel mehr an Bedeutung gewonnen, als ich den Caminho [do Sertão] als Führer machte. Dort habe ich gesehen, dass dieses bescheidene Wissen, über das mein Vater, mein Großvater und mein Onkel sprachen, von einer Wurzel, einer Blume, von etwas, das gut ist für die Gesundheit, oder für die Tiere, oder kunstvoll, irgendsowas, für mich war es etwas, dass ich besaß, für mich behielt, mit meinen Nächsten teilte, aber nie sah, dass es einen solchen Wert hatte. Ich sah, dass sie es wahrnahmen, fassten und verstanden, aber ich weiß nicht, ob es für sie einen Wert hatte. Als dann jemand da aus Rio Grande do Sul oder aus São Paulo kam, der noch nie einen Samen gesehen hatte, der das, das und das macht und ich es ihm erklärte, ein Früchtchen, dass die Mädchen als Spielzeug oder als Zierde, oder alles mögliche andere Zeug, was auf dem Weg immer vorkommt, denn manchmal findet er im Juli statt und manchmal im Januar und jede Jahreszeit hat eine andere Besonderheit. Im Januar sind es die Früchte des Cerrado, es gibt diese Frucht «grão de galo», die großen Erfolg hatte, den Jungs des Trupps aus São Paulo schmeckte sie sehr gut,

65 Auszug aus dem Interview von Almir Paraka (wie Anm. 27). 
und dadurch fühlte ich mich wertgeschätzt mit dem bisschen, dass ich über meine Region wusste und es hat mich dazu veranlasst, mehr zu suchen, die Älteren zu besuchen, die das ein oder andere kannten und so bin ich zum inoffiziellen Forscher meiner Sache geworden, indem ich mir immer mehr Details gemerkt habe. [...] Und als ich da wanderte und diesen alten, den einfachsten Herren der Welt gesehen habe, wie er Gelehrte und Doktoren unterrichtete, Personen mit Diplom aus der akademischen Welt dachte ich: «Warum muss ich mir ein Papier ausstellen lassen, um Gelehrter zu sein? Der Mann ist schon Doktor aller seiner Sachen, die er in seinem Garten hat.» Ich verstand, dass diese Leute, denen wir keinen Wert zusprachen, weil Bücher und andere Dinge wichtiger waren, dieser Mann dort bereits die fertige und organisierte Enzyklopädie war, die wir aufsuchen konnten, man brauchte nur den guten Willen, das zu verstehen, einen Blick dafür zu haben, aber die Jungen verlieren im Allgemeinen diesen Blick für ihre Vorfahren, für diejenigen, die noch am leben sind, für das Werk der anderen. Denn, wenn du nicht weißt, wo du herkommst, woher willst du wissen, wohin du gehst? Du bist hier, eingebunden in diesen Ort, aber du bist kein Teil von ihm, du bist nur hier, wie einer auf der Durchreise, ein Besucher deiner Heimat. Wer das nicht sucht, diese Wurzel, verliert viel. ${ }^{66}$

Wir beobachten in der Aussage einige Nuancen des für Veränderung sorgenden Erfolges, der aus dem Zusammenwirken verschiedener Initiativen resultiert, die in die Richtung gehen, die wir ausgeführt haben. Bezüglich der Wahrnehmung und Offenheit für die Wertschätzung von Volks-, nicht-Gelehrten-Wissens sowie der Wertschätzung des Lebens in Verbindung mit der natürlichen Umwelt aus Almir Parakas Perspektive auf die rosanische Literatur, kommentiert er:

Rosa arbeitet mit der Aufwertung einiger anderer Instanzen und der Produktion von Wissen, das sich vom Hegemonialen und Konventionellen unterscheidet, und stellt dann dieses einfache Leben des Sertanejo wieder her, die Weisheit eines in die Umgebung eingebundenen Lebens, eines in die Umwelt integrierten Lebens. ${ }^{67}$

Die Lektüre ist sehr kohärent in Bezug auf die Literatur des Schriftstellers aus Minas, der solche Merkmale gerade durch die Anerkennung und das Nähren der bedeutenden Weisheit und Kultur des Volkes zeigt, eine Quelle, zu der er zurückkehrt und sie als Mittel zur Erregung der Aufmerksamkeit für einen solchen Reichtum nutzt, der seit jeher dort präsent ist. Doch in Bezug auf diese Interaktion und den Nachhall der sertanejo-Kultur durch das rosianische Werk, das in diesem Sinne als Treibkraft für die Entwicklung einiger Initiativen und Projekte in der Region fungiert, hebt Almir Paraka hervor: «Der Versuch der Aneignung eines literarischen Werkes und seiner Einbeziehung in die Bemühungen um soziale und

66 Auszug aus dem Interview von Fidel Carneiro in Arinos, Sertão in Minas, am 24.1.2017.

67 Almir Paraka: Interview (wie Anm. 21). 
nachhaltige Entwicklung, all das ist sehr neu.» ${ }^{68}$ Sehr vernünftig kommentiert er später in seinem Interview:

\begin{abstract}
Eigentlich ist was wir tun ein Versuch, ich habe bisweilen Angst, sehr signifikante Fortschritte oder Gewinne zu präsentieren. Ein großer struktureller Gewinn, dass können wir vielleicht aus dieser Perspektive der Erweiterung dieser Anstrengung heraus sagen. «O Caminho do Sertão» fügt sich in größere Bemühungen ein, in dem Maße, wie der «Caminho do Sertão» sich selbst festigt, konsolidiert, beginnt das Gespräch, der Dialog mit den staatlichen Sektoren, sei es auf kommunaler, Landes- oder Bundesebene, die beginnen, mit den Organen internationaler Kooperationen zu diskutieren und einen Dialog mit dem brasilianischen Denken mit der Akademie und auch mit sozialen Bewegungen, dem politischen Aktivismus anzuregen. Es beginnt, sich in den Köpfen der Menschen, die an diesem Projekt teilnehmen, die Idee einer freien Schule des Urucuia zu entwickeln, einer Schule des Denkens, mit einer Konzeption, die in diesen speziellen Kontext eingebunden ist, sich von Rosas Werk nährt und von den Werken vieler Autoren, die es erlauben, mit ihnen dieses Bild des Sertão und aller Dinge, die sich um ihn drehen, zu assoziieren, dass Rosa selbst ausgearbeitet hat, sowie andere Bilder, die vielleicht im Laufe der Zeit von anderen Autoren zum BildKonzept des Sertão zusammengefügt und dies hier ausgearbeitet, versucht, experimentiert wird, dass man versucht, in Prozessen anzuwenden, die eigens der «Caminho do Sertão» sind. ${ }^{69}$
\end{abstract}

In diesem Sinne ist es offensichtlich, dass das fragliche Projekt ebenso wie die anderen in Bezug auf seine komplexe und sich in ständiger Transformation befindende Dynamik erörtert wird, das sich entsprechend den Einstellungen und Perspektiven der beteiligten Personen und Einrichtungen auf direkte oder indirekte Weise gestaltet. Wie auch José Idelbrando kommentiert,

Es passiert viel. Offensichtlich geschehen viele dieser Dinge in einer nachvollziehbaren Dimension, was von São Tomé kaum wahrgenommen wird [...] Es handelt sich um immaterielle Errungenschaften wie menschliche Entwicklung, neue soziale Akteure, die Übernahme einer Hauptrolle, das Selbstwertgefühl, das sind einige Errungenschaften, die wir messen können. ${ }^{70}$

Fakt ist, dass das Werk von Guimarães Rosa, sowie das eines jeden anderen Künstlers mit einer solchen Repräsentativität und Resonanz auf nationaler und internationaler Ebene, Raum schafft für seine Ausnutzung als Warenfetisch, und im betreffenden Fall wegen seines direkten Zusammenhangs mit der Verräumlichung der Campos Derais. Das Gebiet setzt sich aus dem reichen Biom des Cerrado zusammen, und schafft Raum für einen kommerziellen Tourismus, der große

68 Ebda.
69 Ebda.
70 Ferreira de Souza: Interview (wie Anm. 27). 
räuberische Ausmaße annehmen kann, vor allem, wenn er von Personen/Agenturen von außerhalb der Region unternommen wird, die nur an der finanziellen Rendite interessiert sind, ohne dem öko-soziokulturellen Einfluss, den dies verursachen kann, die notwendige Sorge zu tragen. In diesem Fall würde die Nutzung des Potenzials von Rosas Literatur bewirken, dass der Raum des Sertão und seine Kultur, die so sehr vom Autor bewundert werden, sehr schnell ihre grundlegenden identitären Charakterzüge verlöre, in dem sie Werte und Muster absorbieren würden, die ihnen nicht eigen sind. Im Dialog mit den Führern und einigen direkt in die Initiativen und Projekte Involvierten zeigt sich die Wahrnehmung dieser Problematik als präsent, wie man im Interview mit Fidel Carneiro feststellen kann, als er bewusst über die Herausforderungen bezüglich der positiven Resultate der Implementierung einer dauerhaften Route im Projekt «O Caminho do Sertão» spricht:

Es gibt wichtige kulturelle Auswirkungen und die müssen berücksichtigt werden, man muss schauen, welche Art von Tourismus wir in unserer Region umsetzen werden, denn sobald Sie die Route festlegen, sie dauerhaft und konstant machen, sie markieren, werden unabhängige Menschen und Gruppen kommen, dann werden diese großen Unternehmer kommen und vielleicht ist das nicht das, was die Gemeinden wollen, das ihnen ein absurder Konsumismus vermittelt wird, der viele Menschen bringen wird. In einer unserer Vorbereitungen für die Führer gingen wir nach São Jorge und dort bemerkte ich diesen Kontrast. Die Leute dort verzweifeln manchmal, dort wohnen 500-800 Menschen, und dann kommen 10.000 Menschen und nach einer Woche verschwinden alle wieder, und was sind die Auswirkungen? Der Müll, die optischen Auswirkungen [...], sie haben keinen direkten Zugang zur Verwaltungseinheit, die ihren Bezirk regiert, ich weiß nicht, ob ich das will. Sie haben Brasília nach São Jorge ${ }^{71}$ verlegt, so verliert sich der Sertão, weil auch die Leute dorthin gehen werden, das wird zum Ziel der Bevölkerung, die Natürlichkeit darf nicht verloren gehen. Ich weiß, dass mehr Leute kommen müssen, ich weiß, dass es notwendig ist, damit die lokale Bevölkerung wächst. [...] Sich darauf einzulassen, ist schwierig, ich habe mich immer darum gesorgt, es hat zwar schon seinen Grund, aber es ist zu schön für uns um zuzusehen, wie das weggeworfen wird. Du siehst ein hübsches Häuschen, das handverputzt wurde mit Sand aus einem Ameisenhaufen, und wenig später reist der einer alles ab und baut Ziegel dahin, und, wo ist es? Zu viel Geld zerstört manchmal, nicht nur manchmal, sondern immer, und Macht ist schrecklich, ob wegen des Geldes oder einer anderen Form von Macht, es ist kompliziert. $^{72}$

71 Ein von Garimpeiros (nach Bodenschätzen Suchende, Anm. d. Üb.) gegründetes Dorf, die auf der Suche nach Quarz in die Region gekommen sind, es hat etwa 700 Einwohner und liegt zwischen dem Tal des Flusses Preto (wo sich der Nationalpark Chapada dos Veadeiros befindet) und dem Tal des Flusses São Miguel. Verfügbar unter http://www.guiaaltoparaiso.com.br/so-jorge. Letzter Zugriff: 10.2.2018.

72 Fidel Carneiro: Interview (wie Anm 66). 
Die Problematisierungen mit diesem Anklang zeigen die Reife und das Wissen um die Gefahren und Herausforderungen dieser Wanderungen, genauso wie die Potenziale und Leistungen, die sich am Horizont abzeichnen, je nachdem, wie die Aktionen durchgeführt werden. Wie bei jeglichem kollektiven Handeln gibt es immer einen Wettbewerb zwischen den fraglichen Interessen. Über diese Art von Problemen kommentiert Damiana Campos im Interview:

\begin{abstract}
Nur, damit Sie einen Widerspruch sehen: Es gibt hier eine Tankstelle namens «Sertão» von einer Person die einen Großteil des Cerrado zerstört hat, weil sie auch mit Gras handelt. Sie haben hier eine Immobilienfirma namens «Sertão», die problematische Landverkäufe im Sertão macht. Aber auf der anderen Seite haben Sie das Projekt «Manuelzinho-da-crôa», das die Konfrontation sucht, Sie haben eine Schule namens «Guimarães Rosa», Sie haben eine Parkstraße. Das ist ein Kampf von uns und von zwölf weiteren Gemeinden. Wir versuchen zu erreichen, dass diese Parkstraße den Namen Guimarães Rosa-Park erhält, was wir auch erreichen werden. Die Landstraße heißt Guimarães Rosa, aber wir wollen, dass die gesamte Straße nach Guimarães Rosa benannt wird. Es gibt also für jeden etwas, der an diesem Strang mitzieht und glaubt, dadurch etwas bewirken zu können [...], das ist uns klar. Das Instituto Rosa e Sertão ist sich darüber ziemlich im Klaren und wir werden dies als eine Strategie für Wandel anwenden. ${ }^{73}$
\end{abstract}

Unter diesen Umständen sind die Bemühungen aller Beteiligten, die sich für Veränderungen einsetzen, die zu erheblichen Verbesserungen in der Region führen, bewundernswert und einer respektvollen Anerkennung wert. Die vor Ort gemachten Erfahrungen und der Kontakt mit den Leitern und anderen in solche Kulturund Umweltprojekte Involvierten haben gezeigt, dass aufgrund der Art und Weise, wie die Aktionen im Laufe der letzten Jahre in die Wege geleitet wurden, ebenso wie aufgrund der Ernsthaftigkeit und des Engagements das immaterielle Potenzial des rosianischen Werts im Bewusstsein über die stets präsenten Gefahren und Schwierigkeiten positiv genutzt wurde, und in dieser Hinsicht können insbesondere während des letzten Jahrzehnts bedeutende Fortschritte verbucht werden. Wie Silva betont, «wird anerkannt, dass die Absichten der Organisationen der Region zu Gute kommen. Es wird angenommen, dass in der Tat auch Expansionen auftreten, die zur politischen und wirtschaftlichen Entwicklung beitragen.» ${ }^{74}$ Die Wissenschaftlerin erklärt darüber hinaus, dass

es sich um eine noble Arbeit handelt; die Unterstützung für soziale Bewegungen sucht und die Aufwertung der urucuianischen Kultur, indem sie die Entwicklung des Tals und das Verständnis der lokalen Identität fördert. [...] Ein solcher Körper trägt zur Erzeugung von Sub-

73 Damiana Campos: Interview (wie Anm. 34).

74 Silva: Nesta água que não para, S. 59. 
jektivität bei den Subjekten und zum Aufbau der kollektiven Vorstellung in Bezug auf das Werk von JGR bei, wodurch er das Leben der Menschen verändert. ${ }^{75}$

In diesem Sinne ist die Wahrnehmung der Art und Weise, wie das durch die rosianische Literatur erzeugte Potential auf Projekte zurückfällt, die zur Förderung von Kultur und nachhaltiger Entwicklung der Region beitragen, sensibel, was den Erhalt von Merkmalen lokalkultureller Ausdrücke ermöglicht und als Strategie zur Stärkung für die Vorschläge zu Bewusstmachung und Erhaltung der regionalen Umwelt funktioniert. Wie Almir Paraka erklärt,

gibt es für uns heute, die wir in gewisser Weise vom sozio-ökologischen Standpunkt aus arbeiten, auch viel Aktivismus in der Region. Von den Gewässern des Urucuia-Flusses, des Urucuia-Einzugsgebiets, zu sprechen, heißt auch, von den Konflikten zu sprechen, die mit Wasser verbunden sind, die leider bereits überall in der Region, im ganzen Urucuia-Einzugsgebiet präsent sind, Konflikte aufgrund der Wassernutzung, die permanente Aggression durch Angriffe auf die Quellen, die im Allgemeinen in den Chapadas geschieht, mit den Umleitungen, die außerhalb der staatlichen Kontrolle liegen. Es ist nicht so, dass der Staat ist nicht darum kümmert, Tatsache ist jedoch, dass der Staat nicht kontrolliert, er hat definitiv keine Kontrolle, die Aufzeichnungen sind nicht zuverlässig und er hat keine permanente Kontrolle über die Wasserwirtschaft. Dies ist ein wichtiges Thema, das wir berücksichtigen müssen. ${ }^{76}$

Immer noch im Sinne der positiven Veränderungen, die im Einklang mit dem Potential der rosianischen Literatur erreicht wurden, ist die Beziehung zu den ökologischen Parks wie dem Nationalpark Grande Sertão Veredas oder dem Staatspark Serra das Araras mit dem Dorf Sagarana erwähnenswert, das ausschlaggebender Ort für die hier aufgeführten sozialen und kulturellen Umgestaltungen ist und das seit Oktober 2003 als Estação Ecológica Estadual (staatlichökologische Station) gehalten wird, die durch das decreto da LEI № 22.897 (dt. Gesetzeserlass Nr. 22.897) vom 11. Januar 2018 als Parque Estadual de Sagarana (dt. Staatspark von Sagarana $)^{77}$ anerkannt wurde und so eine weitere Fortschrittsbewegung für den Erhalt des Cerrado-Bioms in der Region darstellt.

In Bezug auf die angesprochene Pendelbewegung der Einflüsse des Sertão in Minas auf die Produktion der rosianischen Literatur sowie des immateriellen Erbes und dem dadurch erzeugten Potential für transformative Initiativen im Sertão von Minas, so nimmt es auch Silva wahr,

75 Ebda., S. 49.

76 Interview mit Almir Paraka vom 23.1.2017, wörtlich aufgenommen in den Gewässern des Riberão São Miguel.

77 Vgl. Seite 1 des Amtsblatts des Staates Minas Gerais (DOEMG). Verfügbar unter https://www. jusbrasil.com.br/diarios/173599242/doemg-executivo-12-01-2018-pg-1. Letzter Zugriff: 30.1.2018. 
ist nicht bekannt, dass dieses Phänomen im brasilianischen Kontext mit einer anderen Art von Literatur auf solch dialektische Weise geschieht: Die Region inspiriert die poetische Kreation von JGR, JGR dient als Orientierung für politische und kulturelle Aktionen in der Region und ratifiziert diese; Mythos und Raum wandeln sich und aufgrund dieser Dynamik des Austausches entstehen soziale Entfaltungen. ${ }^{78}$

Für die weite Region des Sertão und für die kurze historische Distanz zur rosianischen Literatur befinden sich die deutlich wahrnehmbaren soziokulturellen Veränderungen noch immer im Anfangsstadium und weiterhin offen ist die Definition der Art und Weise, wie diese Veränderungen sich künftig entfalten werden. Es ist jedoch eine Tatsache, dass die rosianische Literatur, die von diesem Sertão viele ihrer Anreize für ihre Produktion erhalten hat, dort nachhallt als eine Spur, die die intensive Verbindung, den unwiderruflichen Pakt, zwischen Rosa und dem Sertão festigt und bekräftigt.

78 Silva: Nesta água que não para, S. 57. 
